\title{
NEVIS CYGLOTRON LABORATORIES
}

\author{
SEARCH FOR THE B-DECAY OF THE PION
}

S. Lokanathan and $J$. Steinberger

\begin{abstract}
COUNMBIA UNIVERSITY PHYSICSITEPARTMENT

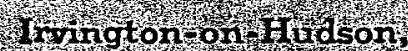

New 1 orl

Joint ONR - AEC Program

Office of Naval Research Contract

Contract N6.ori 110 Task No. I 
Nevis Cyclotron Laboratories

Columbia University

Department of Physics

SEARCH FOR THE B-DECAY OF THE PION

S. Lokanathan and J. Steinberger

$$
\text { CU-81-55-0NR-110-1-Physics }
$$

March, 1955

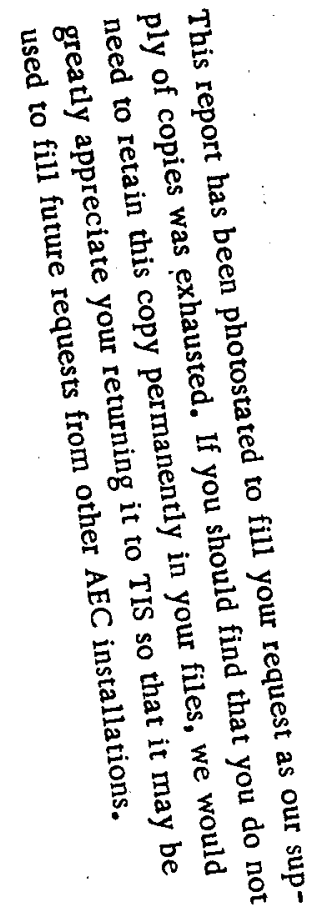




\section{DISCLAIMER}

This report was prepared as an account of work sponsored by an agency of the United States Government. Neither the United States Government nor any agency Thereof, nor any of their employees, makes any warranty, express or implied, or assumes any legal liability or responsibility for the accuracy, completeness, or usefulness of any information, apparatus, product, or process disclosed, or represents that its use would not infringe privately owned rights. Reference herein to any specific commercial product, process, or service by trade name, trademark, manufacturer, or otherwise does not necessarily constitute or imply its endorsement, recommendation, or favoring by the United States Government or any agency thereof. The views and opinions of authors expressed herein do not necessarily state or reflect those of the United States Government or any agency thereof. 


\section{DISCLAIMER}

Portions of this document may be illegible in electronic image products. Images are produced from the best available original document. 


\author{
-1 . \\ Search for the B-decay of the Pion*
}

S. Lokanathan and J. Steinberger

Columbia University, New York, New York

March, 1955

This research was supported by the Joint Program of the Atomic.

Energy Commission and the Office of Naval Research.

\section{ABS TRACT}

An attempt has been made to find the B-decay of the pion. We find for the ratio $\frac{\pi^{+} \rightarrow \mathrm{e}^{+}+\psi}{\pi^{+} \rightarrow \mu^{+}+\psi}=(-.3 \pm .9) \times 10^{-4}$. It is concluded that it is improbable that $B$-decay accounts for more than 1 in $16,000 \pi$ decays.

In an appendix we present the results of some Monte Carlo calculations which were employed in the analysis of this experiment. These calculations give the ranges of electrons with energy near the critical energy. 


\section{INTRODUCTION}

\section{A. Theoretical}

$i_{i}$ Normally the charged pion decays into a muon and light neutral particles, usually assumed to be the neutrino. The possible competing decay into electron and neutrino is not without interest, and we recall here some theoretical points:

a) Connection with nuclear B-decay.

Yukawa postulated the meson $B-$ decay in a two step theory of nucleon B-decay: This hypothesis falls on the one hand because the transition rate of pion-electron decay if non-zero, is at any rate too small to account for the nuclear B-lifetimes, and on the other hand because the observed properties of $B$-decay require Fermi couplings $^{1}$ which are not a consequence of a two step theory with

1 See for instance, S. J. Wu, Proceedings of the 1954 Glasgow Conference on Nuclear and Meson Physics. Pergamon Press, 1955.

pseudoscalar mesons.

The argument may be reversed, and it may be supposed that the pion can transform into an intermediate nucleon-antinucleon pair which annihilates with normal $B$-decay: (1) $\pi^{+} \rightarrow p+\bar{N} \rightarrow e^{+}+\nu$. The pion being pseudoscalar, this transition is forbidden except in pseudoscalar and axial vector $B$-coupling theories. It may then be recalled that it is possible to account for the bulk of $B$-decay data using only scalar and tensor interactions. 1 The smallness of $\pi$-electron decay is therefore not in conflict with experiment. It is however in principle possible to learn about the possible
0391003
page two 
role of pseudoscalar coupling in B-theory from pion-B-decay. Unfortunately the computation of process 1 not only involves divergent Integrals, but different cutoff procedures have led to transition probabilities which differ by several orders of magnitude. 2,3

\section{2 \\ M. Ruderman and R. Finklestein, Phys. Rev. 76,1458 \\ (1949) \\ 3 \\ J. Steinberger, Phys. Rev. $\underline{76}, 1180$ (1949)}

b) Symmetrical coupling of pion to muon and electron.

If the pion were coupled symmetrically to muon and electron, either directly, or by means of an intermediate nucleon anti-nucleon pair, then the relative transition probability $\frac{\pi \rightarrow e+v}{\pi \rightarrow \mu+v}$ de pends only on kinematical factors (masses), field theoretical uncertainties cance1. One than obtains ${ }^{2,3}$ for pseudoscalar coupling of the fermions

$$
\frac{\pi \rightarrow e+\nu}{\pi \rightarrow \mu+\nu}=\left[\frac{M^{2}-M_{e}^{2}}{M_{\pi}^{2}-M_{\mu}^{2}}\right]^{2}=5.4
$$

and for axtal vector coupling

$$
\frac{\pi \rightarrow e+\nu}{\pi \rightarrow \mu+\psi}=\left[\frac{M_{\pi}^{2}-M_{e}^{2}}{M_{\pi}^{2}-M_{\mu}^{2}}\right] \cdot \frac{M_{e}^{2}}{M_{\mu}^{2}}=\frac{1}{8,000}
$$

The pseudoscalar result is roughly equal to the ratio of phase space. It favors $\pi \rightarrow$ e decay and is we11 known to be wrong. The axtal veçtor coupling however discriminates strongly against low mass particles. If it could be established experimentally that the 
ratio $\frac{\pi \rightarrow e}{\pi \rightarrow \mu}$ is indeed less than $1 / 8000$, it would be possible to rule out the possibility of symmetrical coupling of the pion to the muon and electron.

\section{B. Experimental}

In previous attempts to find this decay, ${ }^{4}$ photographic plates

\section{H. L. Friedman and J. Ralnwater, Phys. Rev. 84, 684 (1949)}

were exposed to well collimated meson beams close to the cyclotron target which was bombarded by high energy protons. It was concluded as unlikely that the electron decay of the pion should account for more than one in a thousand pion decays. Recently external meson beams of reasonable intensity have made $1 \mathrm{t}$ possible to use counters as detectors and obtain greater sensitivity. The pion beam is stopped in absorber and the decay electron detected in a counter telescope.

The chief experimental problem is that of distinguishing between $\pi$-decay electrons and $\mu$-decay electrons. This is accomplished by making the detector sensitive to the shorter iffetime and higher energy of the $\pi$-decay electron.

\section{EXPER IMENTAL PROCEDURE}

The experimental arrangement is shown in $F 1 g .1 . \quad$ The $60 \mathrm{Mev}$ $\pi^{+}$beam of the Columbia University Nevis Cyclotron is collimated and monitored by counters \#1 and \#2. Counter \#1 1s a plastic scintillator 4-1/2 inches in diameter and 3/8 inches thick and counter \#2 is a stilbene crystal 2-1/2 inches in diameter and $1 / 8$ 
inches thick, The beam is further collimated by a 2 inch diameter aperture in a 2 inch thick lead shield directly preceding counter \#2 and is slowed by carbon absorbers inserted between $\# 1$ and $\# 2$.

The target is a $1 / 2$ inch thick piece of polyethylene. $\left(1.7 \mathrm{gm} / \mathrm{cm}^{2}\right)$ mounted at approximately $30^{\circ}$ to the incident beam. Of the order of 500 pions stop in the target per second and this represents somewhat more than half of the $1-2$ rate. Of these roughly 1 in 300 will decay with the charged decay product within the acceptance angle of the detector. This detector consists of four plastic scintillators, each 4-1/2 inches in diameter, the first three $3 / 8$ inches thick and the last $1 / 4$ inch thick. The counters $\# 3,4,5$, and 6 are arranged so that it is possible to insert 1 inch thick sheets of absorber between each pair of counters and an additional 6 inches in front of counter $\# 3$.

A block diagram of the electronics is shown in Fig. 2. The following events are recorded:

$M=$ Monitor coincidences $1-2$.

$\mathrm{D}=$ Detector coincidences $3,4,5,6$ with a resolving time of $10^{-8} \mathrm{sec}$.

$M D_{f}=$ 'Fast' coincidences, MD when D occurs within $10^{-7}$ sec after the arrival of $M$.

$M D_{s}=$ 'Slow' soincidences MD when D occurs within $1.8 \times 10^{-6}$ sec after the arrival of $M$.

of the $\pi^{+}$mesons which stop in the target, and decay with a mean life of $2.6 \times 10^{-8} \mathrm{sec}, 5$ the vast majority produce $\mu$-mesons.

5 C. Wiegand, Phys. Rev. 83, 1085 (1951) 
The muons have a range of $2 \mathrm{~mm}$ in the polyethylene, about $1 / 6$ of its thickness. Approximately 95 percent of these $\mu$-mesons therefore stop and decay in the same target piece. The mean-1ife of this decay is $2.2 \times 10^{-6} \mathrm{sec}^{6}$ two orders of magnitude longer than

6 W. E. Be11 and E. P. Hincke, Phys. Rev. 88, 1424

(1952)

the parent process, and results in a continuous B-spectrum with 53 Mev maximum energy. 7

7

See for instance, Sargent, Rinehart, Lederman and Rogers. In Press.

With small absorber thicknesses therefore the events $D$ are due to the $\mu$-electrons. Of these 56 percent $=1-e^{-1.8 / 2.2}$ are expected to be counted with the long gate of $M D_{s}$. Between 4 percent and $3-1 / 2$ percent of these are counted in the short gate, $\mathrm{MD}_{f}$. The rate $D$ may therefore be used to determine the produce of the rate of stopping $\pi^{\prime}$ s multiplied by the acceptance solid angle of $D$. The ratios $M D_{f} / D$ and $M D_{s} / D$ may be used to determine the effective gate width of these channels.

Two sets of observations were made. In run \#1 rates were observed with thicknesses of polyethylene in 1 inch steps from zero absorber thickness (in addition to the counters and target) to 9 inches of absorber. In run \#2 only 3 inches and 9 inches of polyethylene were used. In both runs data were obtained with and without the target, and the bulk of the observation was made with 
9 inches of absorber. This 1atter thickness corresponds to an energy loss for relativistic particles of 55 Mev due to ionization alone. $\mu$-decay electrons cannot penetrate this absorber; however, some are nevertheless detected through the conversion of their bremstrahlung. The geometry of counters and absorbers in $D$ is chosen to minimize this effect. Experimentally we find $1 / 500$ of the decay spectrum detected in this manner. This is a rate which might reasonably be expected for bremstrahlung conversion. The $\mu-$ decay background in $\mathrm{MD}_{\mathrm{f}}$ with 9 inches of polyethylene is therefore approximately $.04 \times 1 / 500=8 \times 10^{-5}$ of the total $\mu$-decay rate.

\section{I . EXPER IMENTAL RESULTS}

The experimental results were obtained in 2 three-day runs and are presented in Tables 1 and 2 and in Fig. 3 .

IV. ANALYSIS OF THE DATA

A) Product $\mathrm{K}$ of stopped meson $\mathrm{flux}$ and detector solid angle.

$K$ is obtained by extrapolating the observed rate $D$ from small absorber thickness to zero absorber thickness, as discussed in the introduction. For run \#1 this can be done using Fig. 3 , and $\mathrm{K}_{1}=$ $3330 \pm 50$ per $10^{6}$ monitor counts. For run \#2 we multiply the rate observed with 3 inches of polyethylene by the ratio of counts D extrapolated to zero absorber to counts $D$ with 3 inches of polyethylene as determined in Fig. 3. This ratio being $2.0, \mathrm{~K}_{2}=$ $2640 \pm 50$ per $10^{6}$ monitor counts.

B) Acceptance time $\lambda$ for the detection of decay positrons in $\mathrm{MD}_{\mathrm{f}}$. 
This can be determined from the counting rates of the $\mu$-decay positrons using small absorber thickness:

$$
\lambda=\lambda_{\mu \rightarrow e} \ln \left(\mathrm{D} / \mathrm{D}-\mathrm{MD}_{\mathrm{f}}\right)
$$

We find $\lambda_{1}=8.6 \times 10^{-8} \mathrm{sec}$ for run $\# 1$ $\lambda_{2}=6.7 \times 10^{-8} \mathrm{sec}$ for run \#2

C) Detection probability of positrons from the decay of $\pi^{+} \rightarrow e^{+}+v$.

These positrons will have an energy of $71 \mathrm{Mev}$, one half of the rest energy of the pion. With 9 inches of polyethylene, the average ionization loss of a minimum ionizing particle is $45 \mathrm{Mev}$ in the $21 \mathrm{~g} / \mathrm{cm}^{2}$ of polyethylene, about $7 \mathrm{Mev}$ in the four detectioncounters $\left(3.5 \mathrm{~g} / \mathrm{cm}^{2} \mathrm{CH}\right)$ and $2.5 \mathrm{Mev}$ in one half of the meson stopping target $\left(1.1 \mathrm{~g} / \mathrm{cm}^{2} \mathrm{CH}_{2}\right)$ for a total loss of $54.5 \mathrm{Mev}$ through ionization. In the appendix we calculate the probability with which electrons of given energies will penetrate absorbers of given iontzation loss taking radiation and multiple scattering into account. From Fig. 4, we interpolate that 71 Mev electrons will be detected with a probability $E=0.48$ when 9 inches of polyethylene are present. However, this will not be quite true in the case of positrons for these can annihilate in flight. This is in large measure balanced by a similar effect on the positrons from the $\mu-e$ decay which serve as calibration. We estimate that this effect reduced the probability $E$ by about 2 to 3 percent and therefore use the value $E=.46$.

D) Correction for the $\mu-e$ decay positrons. The $\mu$-decay positrons which are counted in $\mathrm{MD}_{\mathrm{f}}$ with 9 inches 
of absorber through the conversion of the bremstrahlung radiation are directly determined from the rates $D$ or $\mathrm{MD}_{\mathrm{S}}$ which are almost entirely due to this effect.

Thus the number which has to be subtracted from $\mathrm{MD}_{\mathrm{f}}$ is

$$
\delta=\left(M D_{S}\right) 9 \text { inches } \frac{\left(1-e^{\lambda / 2.2 \times 10^{-6}}\right)}{\left(1-e^{-1.8 / 2.2 \times 10^{-6}}\right)}
$$

or $\delta=$ (D) 9 inches $\left(1-\mathrm{e}^{-\lambda / 2.2 \times 10^{-6}}\right)$

We find $\delta=(.18 \pm .025)$ per $10^{6}$ monitor counts for run \#1

$$
\delta=(.089 \pm .02) \text { per } 10^{6} \text { monitor counts for run \#2 }
$$

E) Correction for inverse photomeson production and charge exchange scattering.

The target is traversed by a $f l u x$ of pions measured in $M$. Only one half of these actually stop in the target, the average energy in the target may be approximately 25 . Mev, and the average thickness of carbon traversed is $\sim 1.5 \mathrm{~g} / \mathrm{cm}^{2}$. The meson has a finite probability for nuclear interaction with subsequent $\gamma$-emission, either from the inverse of photomeson production, or from charge exchange scattering. The former process may be estimated to have a cross section of $2 \pm 1 \mathrm{mb}$ in this energy range, from observations $^{8}$ on the reaction $\gamma+C \rightarrow B^{*}+\pi^{+}$, which shows a certain

J. Steinberger and A. S. Bishop, Phys. Rev. 86, 171 (1952) resemblance to its inverse. The $\gamma$-rays emitted are of the order of $130 \mathrm{Mev}$ and w111 have a detection probability of approximately 
0.35 in $D$. The counting rate due to this effect is therefore approximately:

$$
\begin{aligned}
\mathrm{MD}_{\mathrm{f}} / \mathrm{M} & =(2 \pm 1) \times 10^{-27} \times\left[1.5 \times 6 \times 10^{23 / 12] \times[.049 / 4 \pi] \times .35}\right. \\
& =(2.2 \pm 1.1) \times 10^{-7}
\end{aligned}
$$

The charge exchange cross section in carbon has not been measured, however, it appears to be less than $1 \mathrm{mb}$ for positive plons under $30 \mathrm{Mev} .^{9}$ The effictency for detecting these $\gamma$-rays 1 s some-

9

J. Tinlot, Private Communication.

what less, because of the lower energy; it is approximately 0.2 . The corresponding rate should therefore be

$$
\begin{aligned}
\mathrm{MD}_{\mathrm{f}} / \mathrm{M} & \leq 2 \times 1 \times 10^{-27} \times\left[1.5 \times 6 \times 10^{23} / 12\right] \times[.049 / 4 \pi] \times 0.2 \\
& =1.25 \times 10^{-7}
\end{aligned}
$$

The charge exchange correction is therefore less than one half of the inverse photo process correction, but will not be made, since only an upper limit for this' correction exists.

F) Fraction of $\pi^{\prime} \mathrm{s}$ decaying to electrons.

The net counting rate $M D_{f} / M$ after subtraction for $\mu$-decay electrons and inverse photoprocess is

$$
[(.38 \pm .16)-(.18 \pm .025)-(.22 \pm .11)]=\underset{\text { monitor counts for run }] 1}{(-.02 \pm 21)} \text { per } 10^{6}
$$

and $[(.254 \pi .10)-(.089 \pm .02)-(.22 \pm .11)]=(-.055 \pm .15)$

$$
\text { per } 10^{6} \text { monitor counts for run \#2 }
$$


The fraction of $\pi$-mesons undergoing $B$-decay is

$$
\begin{aligned}
& \left(M D_{f} / M\right)_{\text {corrected }} \times 1 / K \times 1 / E=f \\
& f_{1}=-.13 \pm 1.36 \times 10^{-4} \text { for run } \# 1 \\
& f_{2}=-.45 \pm 1.23 \times 10^{-4} \text { for run } \# 2
\end{aligned}
$$

Combining these two results, our experiment yields the ratio:

$$
\frac{\pi \rightarrow e}{\pi \rightarrow \mu}=f=(-.3 \pm .9) \times 10^{-4}
$$

The quoted error is the standard deviation and includes the statistical uncertainty as well as an estimate of the error in the subtraction for the inverse photomeson production.

It is therefore not likely that the actual $\pi \rightarrow$ e decay fraction is greater than .6 $610^{-4}$ or one in 17,000 . The experiment is approximately twenty times more sensitive than previous attempts to find this decay mode, but no positive evidence is obtained. It seems therefore improbable that the pion is coupled symmetrically to the muon. 
APPENDIX

Straggling of electrons with energies of the order of the critical energy.

In this energy range the straggling is primarily due to radiation and multiple scattering. This problem has not been solved. analytically, although the processes are well understood. We have solved the problem with an accuracy sufficient for our purposes by making Monte Carlo calculations for the radiation straggling and combining these with similar calculations ${ }^{1}$ on the reduction in range due to the irregularity of the trajectory (multiple scattering) chief1y near its end.

The radiation straggling calculations were carried out at 6 energies: $E^{\prime}=25,35,50,70,85$ and 100 Mev. The Bethe-Heitler radiation loss formula is approximated by the form which corresponds to uniform energy loss over the spectrum:

$$
\mathrm{dN}(\mathrm{E}) / \mathrm{dx}=1 / \mathrm{EX}
$$

$N(E)$ is the number of quanta of energy $E$ radiated per untt energy interval and $\mathrm{X}$ is the radiation length; in our case of $\mathrm{CH}_{2}$ this is $65 \mathrm{~g} / \mathrm{cm}^{2}$. The absorber is then divided into sections of 5 Mev lonization 1oss. In $\mathrm{CH}_{2}$ the ionization loss of minimum outgoing particles is $2.18 \mathrm{Mev} / \mathrm{c}^{2}$, so that each section corresponds to .0354 radiation length. The radiation loss probability distribution is then divided into 100 regions of equal probability and two digit random numbers are chosen for each interval. The calculation proceeds by allowing a trial electron to penetrate to the center of 
$\mathbf{z}$

the first section by losing 2.5 Mev through ionization. It then radiates according to the loss picked from the radiation probabil1ty distribution by the random number of the section, loses 5 Mev by Ionization to get to the center of section two, radiates to its luck in this section and so on. We calculate for 100 trajectortes at each of the six energies. The results are tabulated in Table 3. In Fig. 4 these results are plotted after the statistical irregularities are smoothed. In the same figure we also show the results of folding the multiple scattering distribution into these results. 10 The range is given in units of ionization 1oss. For the

10 The multiple scattering affects the trajectory chiefly near the end. We neglect the energy dependence of this straggling, and use the calculations made earlier for the multiple scattering of $50 \mathrm{Mev}$ electrons.

J. Steinberger, Phys. Rev. 75, 1135 (1949)

purposes of the experiment, it is necessary to know the detection probability (probability that the range be in excess) as a function of energy for different absorber thicknesses, and this is plotted in Fig. 5. The data of Fig. 5 are derived from Fig. 4.

We wish to point out here that the results presented in Table 1 and Figs. 1 and 2 may also be used to predict the behavior in other materials, if the energy scale is converted by the factor:

$$
\overline{\mathbf{E}}=\overline{\mathbf{I}} / \mathbf{I}
$$

where $I$ is the ionization loss per radiation length, in this case 


\section{$142 \mathrm{Mev}$.}

The computations have received some confirmation by comparing the observed $\mu \rightarrow e$ range curve with a computation of this range distribution using the calculated electron ranges and a spectrum for the decay electrons given by Michel's parameter $\rho=1 / 2$. $^{7}$ This is shown in Fig. 3 . 


\section{5}

\section{FIGURE CAPTIONS}

\section{FIGURE}

1. Arrangement of counters and absorbers.

2. Block diagram of circuits.

3. Counting rates $M D_{S}$ and $M D_{f}$ as a function of the absorber thickness in the detector $D$, obtained in run \#1. The rates $M D_{f}$ have been multiplied by the factor 25.6 so that the two curves coincide for small absorber thicknesses. The solid curve is the expected range dependence of $\mu$-decay electrons $(\rho=1 / 2)$. The dotted curve is the expected $\pi \rightarrow$ e range dependence, and should be compared with the difference between the experimental curves $\mathrm{MD}_{\mathrm{f}}$ and $\overline{M D}_{\mathrm{S}}$.

4. Smooth curve presentation of the results of the Monte Car1o range calculations: Detection Probability vs. Range in Polyethylene.

5. Smooth curve 1resentation of the results of the Monte Carlo Calculations: Detection Probability vs. Energy in Polyethylene. The range parameter is given in units of MEV ionization loss. 


\section{TABLE 1}

16

\begin{tabular}{|c|c|c|c|c|c|c|c|c|c|c|}
\hline \multirow{3}{*}{ 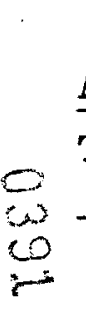 } & Absorber & & & \multicolumn{2}{|c|}{ Target In } & \multirow{2}{*}{$\frac{\text { Target }}{\operatorname{Mx} 10^{6}}$} & \multicolumn{2}{|l|}{ Out } & \multicolumn{2}{|c|}{$\begin{array}{l}\text { Net per } 10^{6} \\
\text { Monitor Counts }\end{array}$} \\
\hline & $\begin{array}{c}\text { Thickness of } \mathrm{CH}_{2} \\
\text { in inches }\end{array}$ & $\begin{array}{c}\text { Total ionization } \\
\text { loss in Mev }\end{array}$ & $\mathrm{M} \times 10^{6}$ & D & $M D_{f}$ & & D & $\mathrm{WD}_{\mathrm{f}}$ & D & $\mathrm{MD}_{f}$ \\
\hline & 0 & 7.5 & 4.26 & 14,453 & 541 & 1.54 & 364 & 1 & 3,164 & 120 \\
\hline E & 1 & 12.5 & 4.10 & 11,964 & 468 & 1.54 & 149 & 1 & 2,811 & 113 \\
\hline- & 2 & 17.5 & 3.07 & 7,510 & 274 & 1.54 & 94 & 3 & 2,389 & 87 \\
\hline & 3 & 22.5 & 3.07 & 5,602 & 212 & 1.54 & 66 & 2 & 1,787 & 68 \\
\hline & 4 & 27.5 & 3.08 & 3,878 & 143 & 1.54 & 37 & 4 & 1,246 & 44 \\
\hline & 5 & 32.5 & 3.07 & 2,318 & 78 & 1.54 & 28 & 0 & 738 & 25.4 \\
\hline & 6 & 37.5 & 3.07 & 1,257 & 45 & 1.54 & 29 & 1 & 392 & 14.1 \\
\hline & 7 & 42.5 & 3.07 & 471 & 23 & 1.54 & 23 & 0 & 139 & 7.5 \\
\hline & 8 & 47.5 & 3.07 & 122 & 3 & 1.54 & 21 & 1 & 26.1 & .33 \\
\hline & 9 & 52.5 & 29.4 & 606 & 15 & 15.5 & 206 & 2 & 7.3 & $.38 \pm .16$ \\
\hline
\end{tabular}


TABLE II

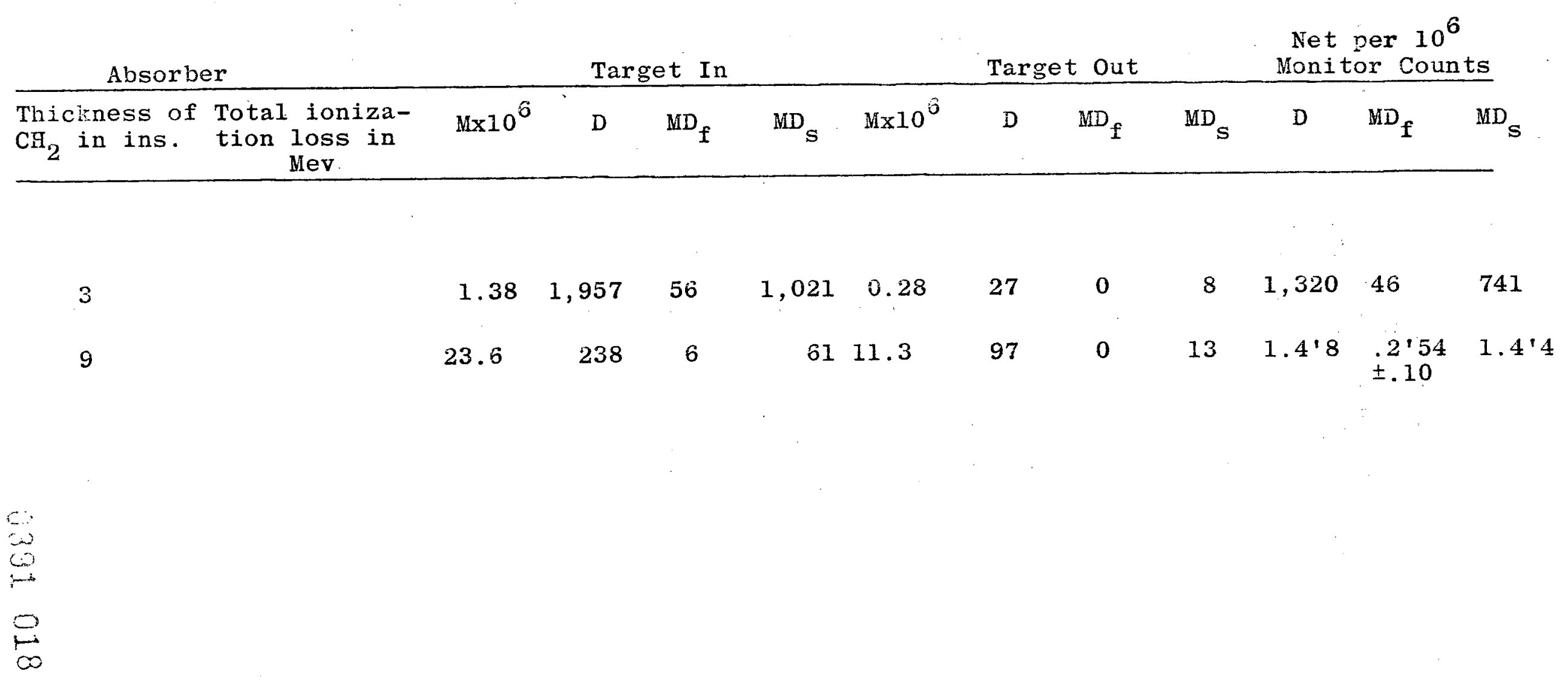


Results of the Monte Carlo calculations on the ranges of electrons in polyethylene. 100 trials are presented for each of six energies. The ranges in the column 'range interval' are given in units of ionization loss in Mev.

\begin{tabular}{|c|c|c|c|c|c|c|}
\hline Interval & 25 & 35 & 50 & 70 & 85 & 100 \\
\hline $0-2.5$ & & & & & & \\
\hline $2.5-.5$ & & & & & & \\
\hline 5. -7.5 & 1 & & & & & \\
\hline $7 \cdot 6-10$ & & 1 & & & & \\
\hline $10 .-12.5$ & 3 & & 2 & & 1 & \\
\hline $12.5-15$ & 3 & & & 2 & 1 & \\
\hline $15-17.5$ & 6 & 2 & & & 2 & 1 \\
\hline-20 & 11 & 5 & 2 & & 1 & 1 \\
\hline-22.5 & 7 & 4 & 1 & & 2 & 1 \\
\hline-25 & 69 & 6 & 1 & & 1 & 2 \\
\hline-27.5 & & 3 & 1 & 1 & 1 & \\
\hline-30 & & 8 & 1 & 5 & 1 & 3 \\
\hline-32.5 & & 6 & 5 & 2 & 1 & 1 \\
\hline-35 & & 65 & 5 & 3 & 1 & \\
\hline-37.5 & & & 6 & 1 & 3 & 1 \\
\hline-40 & & & 5 & 6 & 5 & \\
\hline-42.5 & & & 8 & 2 & 3 & 2 \\
\hline-45 & & & 8 & 4 & 2 & 3 \\
\hline-47.5 & & & 17 & 3 & 3 & 1 \\
\hline-50 & & & 38 & 4 & 3 & 1 \\
\hline-52.5 & & & & 6 & 2 & 1 \\
\hline-55 & & & & 4 & 6 & 1 \\
\hline-57.5 & & & & 6 & 3 & 2 \\
\hline-60 & & & & 8 & 5 & 2 \\
\hline-62.5 & & & & 6 & 4 & 3 \\
\hline-65 & & & & 6 & 4 & 4 \\
\hline-67.5 & & & & 10 & 6 & 3 \\
\hline-70 & & & & $\underline{12}$ & 5 & 5 \\
\hline
\end{tabular}


$\frac{19}{\text { TABLE III (Continued) }}$

E

25

35

50

70

85

100

70-72.5

$5 \quad 5$

72.5-75

5

1

$-77.5$

5

7

$-80$

8

3

$-82.5$

7

5

$-85$

7

3

$-87.5$

6

$-90$

5

$-92.5$

5

$-95$

7

$-97.5$

7

97.5-100

5 


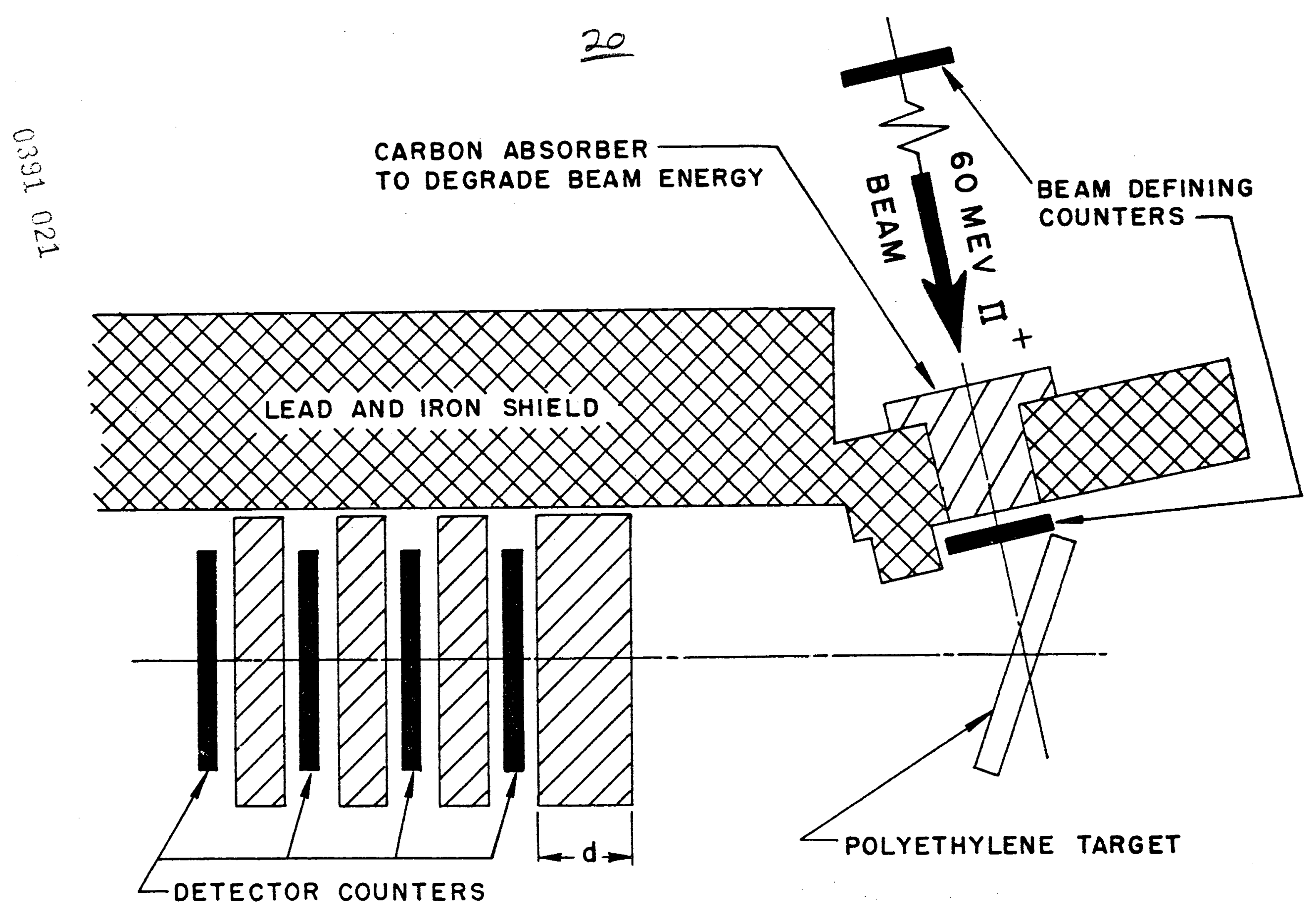


21

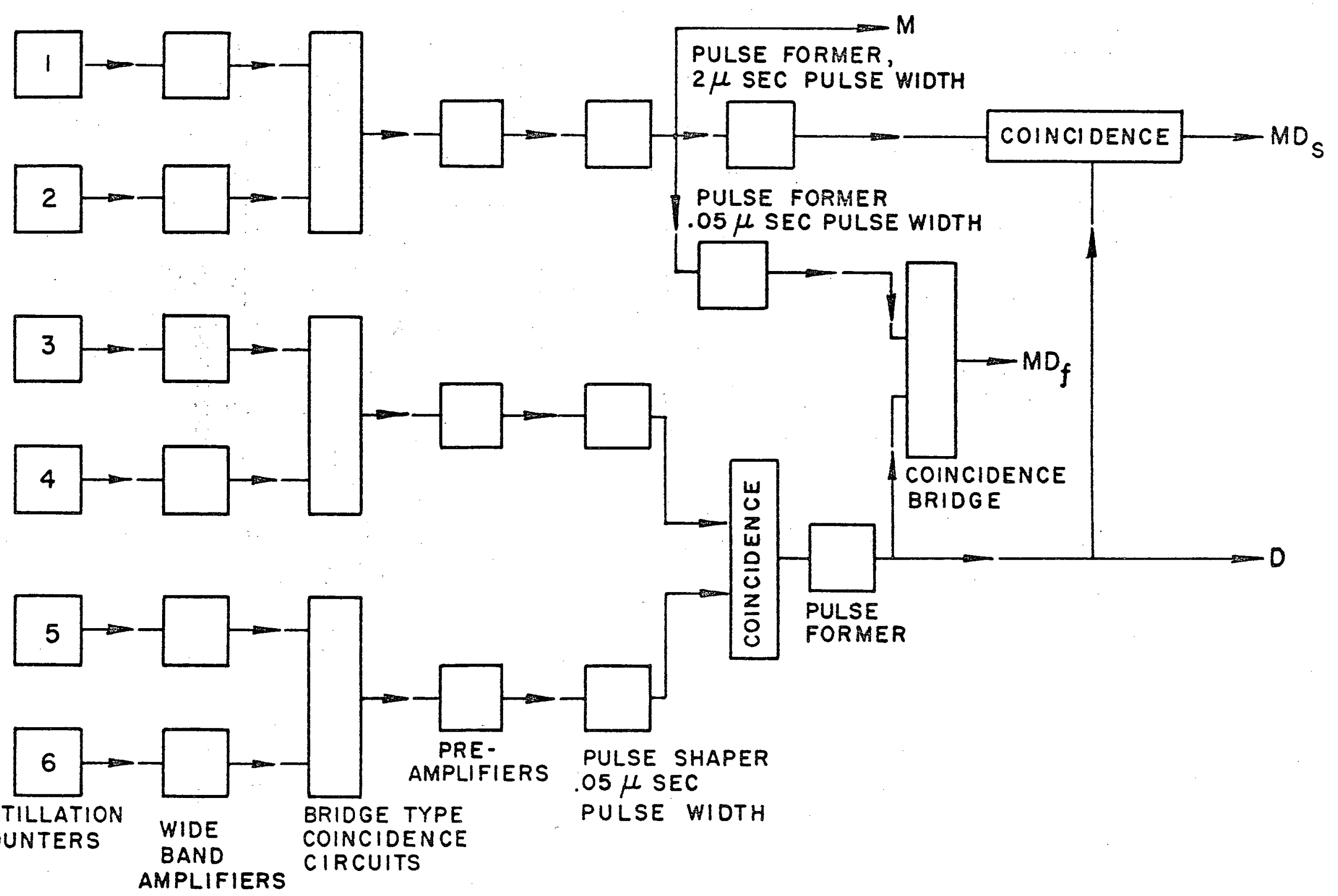


22

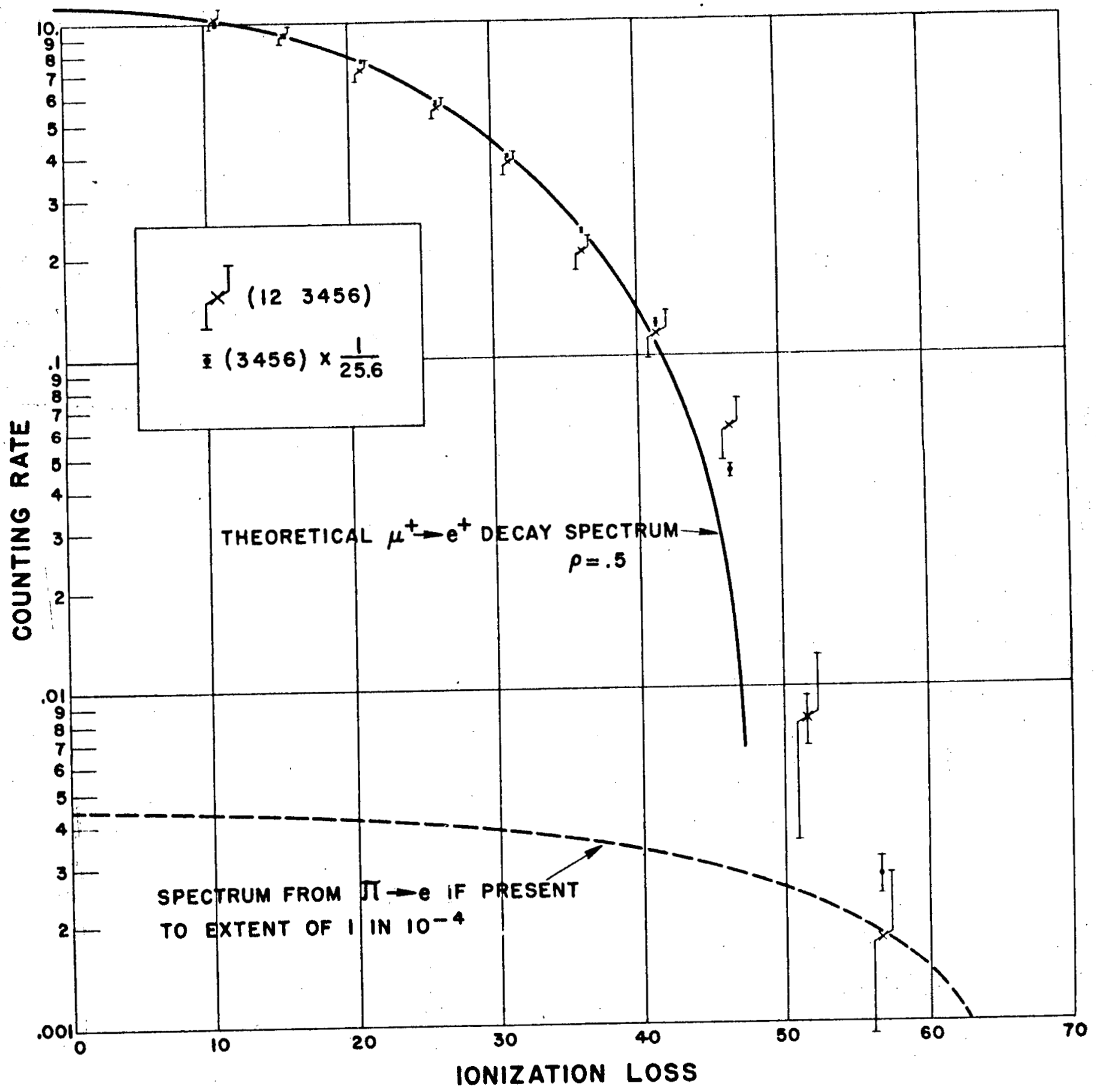

0391023 


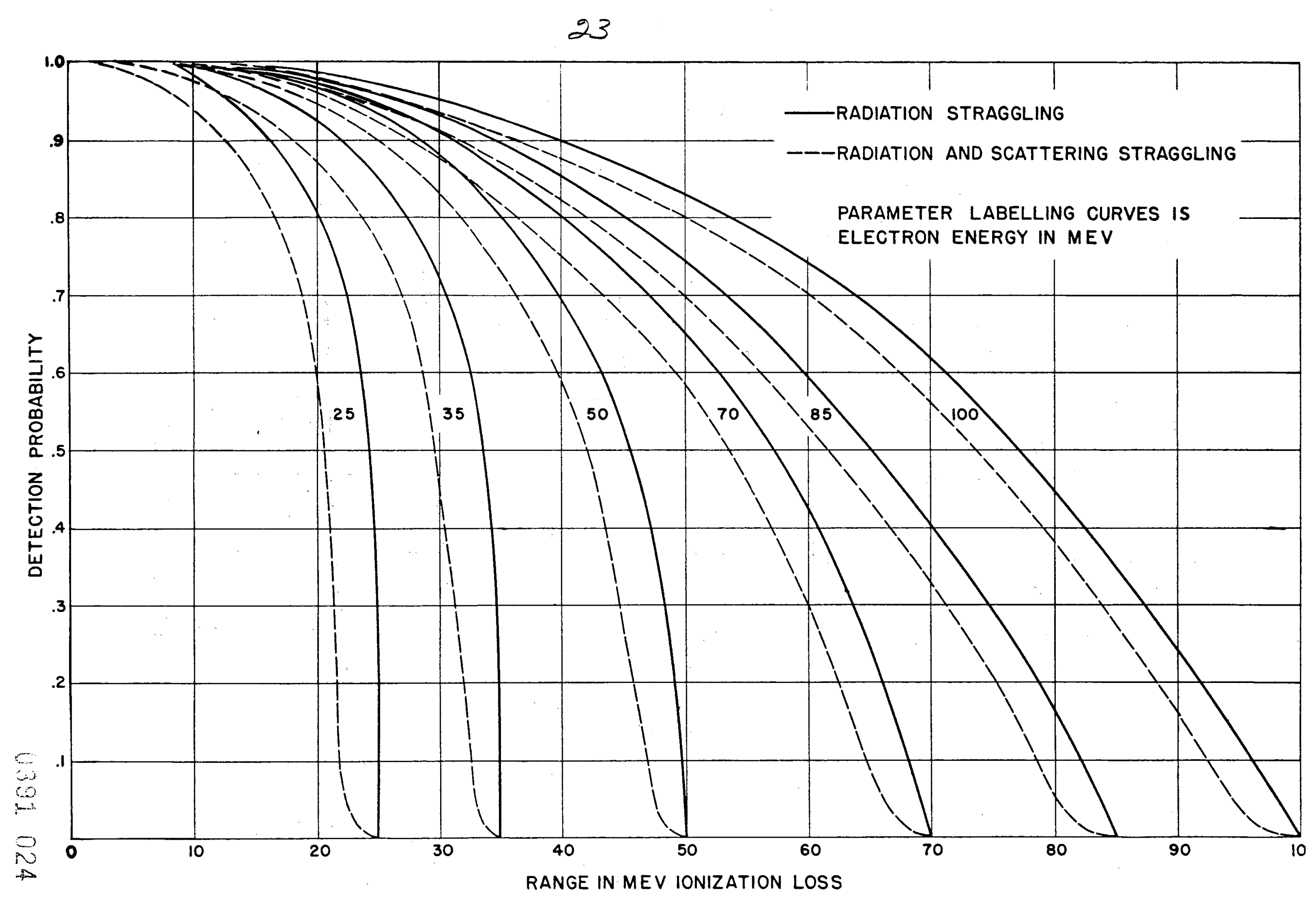




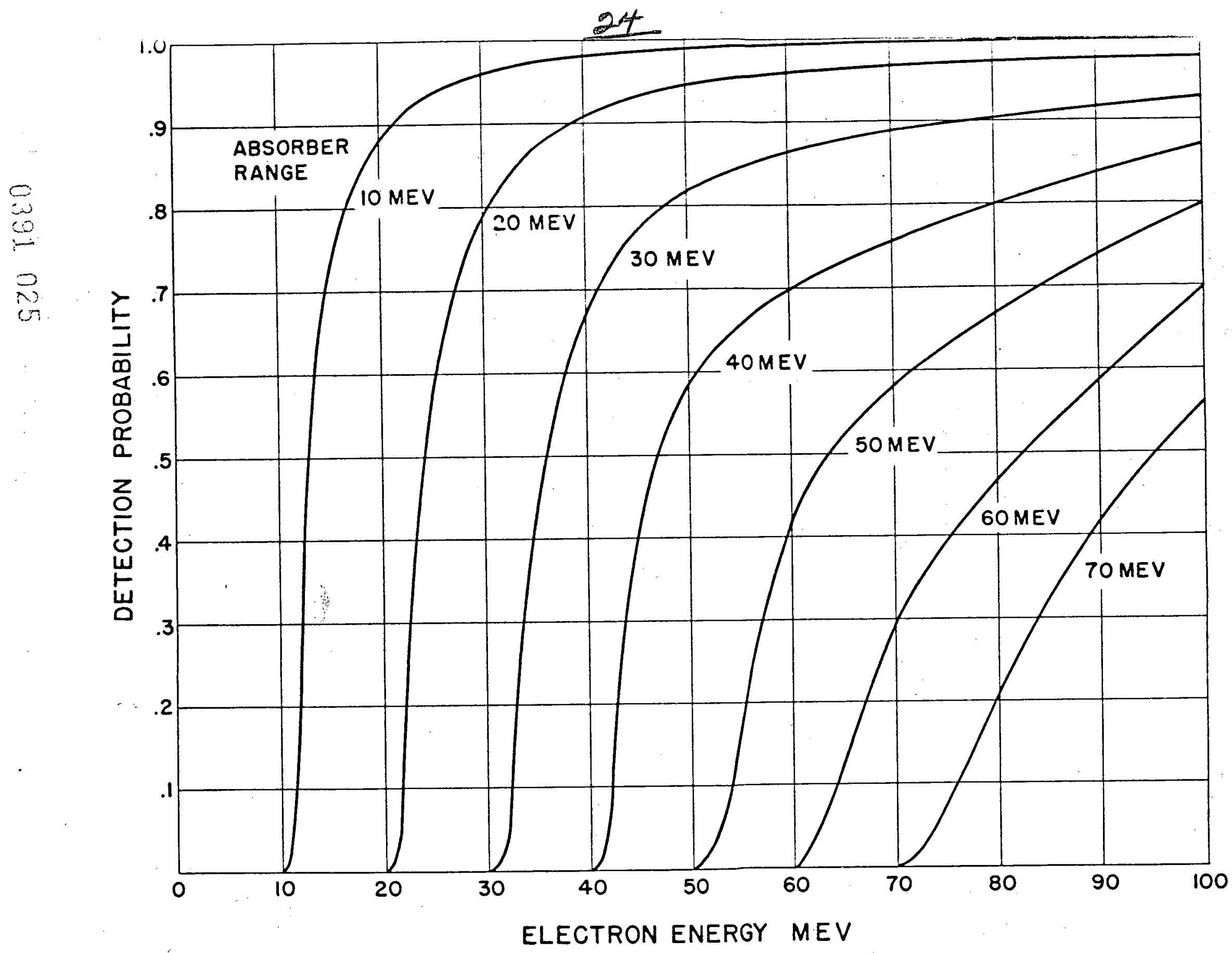

